\title{
Case Report \\ LARYNGEAL TUBERCULOSIS PRESENTING WITH PRIMARY SYMPTOMS OF LARYNGEAL CARCINOMA
}

\author{
Junaid $\mathbf{M}^{1}$, Qadeer $\mathbf{S}^{2}$, Sobani ZA ${ }^{3}$, Haroon $\mathbf{S}^{4}$, Ghaffar $\mathbf{S}^{5}$
}

1 Department of Otolaryngology, Jinnah Medical and Dental College, Karachi, Pakistan

2,3 Department of Head and Neck Surgery, Aga Khan University, Karachi, Pakistan

4 Department of Pathology and Microbiology, Aga Khan University Hospital, Karachi, Pakistan

5 Department of Surgery, King Abdul Aziz Medical City, National Guards Health Affairs, Riyadh, Kingdom of Saudi Arabia

\section{ABSTRACT}

Laryngeal tuberculosis (TB) occurs in about $1 \%$ of patients suffering from pulmonary tuberculosis; however presentation of these patients with primary laryngeal symptoms is a rarity. In such situations it forms a diagnostic dilemma between laryngeal TB and the more common laryngeal carcinoma.

Highlighting this dilemma we present our patient, a 76 year old male, farmer presenting with primary complaints of progressive dysphagia for 3 weeks. This patient happens to be our second case of laryngeal TB, presenting with primary laryngeal symptoms in a span of under 2 years.

We present these cases to emphasize that although laryngeal tuberculosis presenting with primary laryngeal symptoms is a rarity, it must be considered when evaluating dysphagia or dysphonia in populations where TB is endemic.

\section{INTRODUCTION}

Laryngeal tuberculosis (TB) considered to be rare sequelae of pulmonary TB; occurs in about $1 \%$ of patients suffering from pulmonary infections. ${ }^{1,2}$ However presentation of patients with primary laryngeal symptoms such as dysphagia or dysphonia is a rarity.ln such situations it forms a diagnostic dilemma between laryngeal TB and the more common laryngeal carcinoma (LCa). Further compounding this situation ${ }^{3}$ is the similar distribution of risk factors and socio-demographic characteristics that both these diseases thrive in. ${ }^{4}$

Highlighting the aforementioned dilemma we present our second case of laryngeal TB, presenting with primary laryngeal symptoms in a span of under 2 years.

\section{Case}

A 76 year old male, farmer from Karachi, was referred to our clinic by a primary care physician due to progressive dysphagia for the past 3 weeks.
The dysphagia began as intolerance to solid and semi-solid food; which had progressed to the point where he had not been able to tolerate a liquid diet for the past 2 days. Focused history revealed that he had developed gradually progressive hoarseness over the past 6 months. He initially managed the hoarseness with home remedies, including honey and warm water gargles, but this did not seem to alleviate his symptoms. He also developed dyspnea on severe exertion during this period.

On review of systems the patient denied any history of fever, hemoptysis or stridor; however he complained of intermittent bouts of cough which were relieved with medications from a local Hakeem (traditional healer). His family had also noticed a subjective weight loss (he did not have a previously recorded weight for comparison) and fatigability in past few weeks.

The patient did not recall any significant past medical and surgical issues or exposure to tuberculosis (TB). He had an 80 pack year history of smoking, intermixed with the use of water pipes and unfiltered cigarettes. 
On examination he appeared drowsy but arousable. Ear, nose and oral cavity examination was within normal limits. Indirect laryngoscopy revealed pooling of saliva. A fiber optic laryngoscopy was performed which revealed supraglottic inflammation with pooling of saliva and diffuse edema over both false cords. The entire length of the true vocal cords could not be visualized; however they were mobile. Granulations were noted along the right vocal cord and post cricoid area. The patient was immediately admitted for rehydration and planned for direct laryngoscopy with biopsy.

Pre-operative evaluation showed hemoglobin of $8.1 \mathrm{mg} / \mathrm{dl}$, blood urea nitrogen of $44 \mathrm{mg} / \mathrm{dl}$ and creatinine of $1.8 \mathrm{mg} / \mathrm{dl}$. His electrolytes and blood sugar levels were within normal range. Chest X-ray revealed bilateral infiltrates, with multiple cavitatory infiltrates suggestive of active tuberculosis. The patient was immediately shifted to an isolation room and a pulmonology consult was generated.

It was decided to proceed with direct laryngoscopy and biopsy with the addition of bronchoscopy and bronchoalveolar lavage (BAL). Direct laryngoscopy revealed granulations along the arytenoids and post cricoid area along with diffuse swelling over the false cords. The aryepiglotic folds, epiglottis and piriform sinus were devoid of disease. A biopsy was taken from the visualized granulations and sent for Ziehl-Neelsen staining, acid fast bacilli culture, fungal staining, fungal culture and histopathological examination. Bronchoalveolar lavage was also sent for cytology and culture.

Postoperatively the patient was shifted back to an isolation room and started on empiric anti tuberculous therapy (ATT). Ziehl-Neelsen staining of the biopsy sample and cytology of BAL fluid was positive for acid fast bacilli. He was discharged on post-operative day 3 after adequate hydration and a provisional diagnosis of tuberculosis. He was started on ATT and advised monthly follow up visits with transmission precautions at home.

Histopathological examination of the excised tissue revealed giant cells with caseous necrosis, no signs of malignancy were noted (Figure 1). Tissue culture revealed heavy growth of acid fast bacilli.

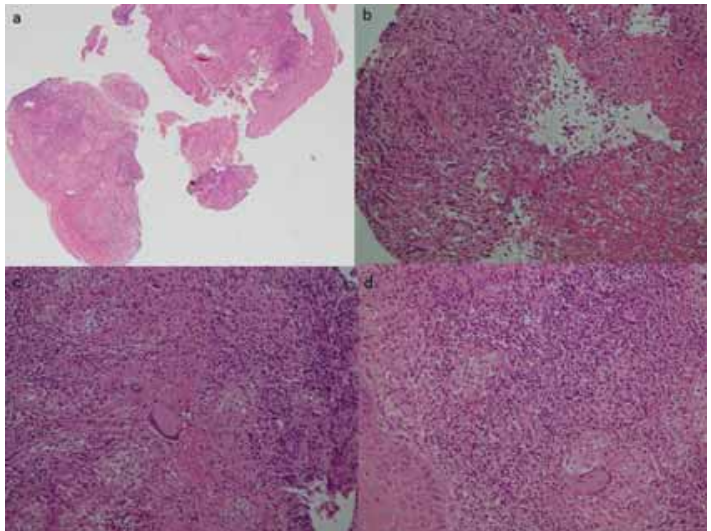

Figure 1. Histopathological examination of laryngeal biopsy specimen, a) hematoxylin and eosin stain at low power showing granulomatous inflammation, b) Foci of necrosis are seen on right lower side, $c$ ) horse-shoe shaped giant cell is seen in the center, d)granulomas comprising of giant cells, epithelioid cells and histiocytes can be visualized.

At one month follow up the patient's dysphagia had resolved. Fiber optic laryngoscopy showed minimal granulation, with persistent edema. No pooling of saliva observed.

\section{DISCUSSION}

Having the $8^{\text {th }}$ highest estimated prevalence of tuberculosis in the world; Pakistan accounts for $44 \%$ of tuberculosis cases in the Eastern Mediterranean Region. ${ }^{5}$ Interestingly the same population has one of the highest age- standardized rates of head and neck cancer an estimated $22.5 / 100,000$ in males and $20.4 / 100,000$ in females. The same database reported that laryngeal carcinoma was the $3^{\text {rd }}$ most common cancer among males overall in the city of Karachi. ${ }^{6}$ Interestingly, both the diseases may have similar presentations, and their risk factors seem to be concentrated in the same population, creating a diagnostic dilemma.

It is hypothesized that laryngeal tuberculosis most commonly occurs due to hematogenous dissemination or direct extension of a primary pulmonary TB infection. Considering this hypothesis to be valid most patients with laryngeal tuberculosis should present with signs and symptoms of their primary pulmonary TB. However patients may present without manifestations of pulmonary TB with complaints of dysphagia and/ or dysphonia. 
Highlighting the aforementioned dilemma, we present our second case of laryngeal TB, with characteristic presenting features of LCa. Our first patient described earlier was a 40 year old male, presenting with persistent hoarseness and weight loss; without associated fever, night sweats, cough or dysphagia. The previous patient did not have any history of tobacco or irritant use; however considering the absence of associated symptoms and characteristic history he was initially worked up for laryngeal carcinoma. ${ }^{4}$

This patient as well presented with progressive dysphagia, hoarseness and dyspnea on exertion. Although he did not have any fever, hemoptysis or stridor; he complained of intermittent bouts of cough which were relieved with medications from a local Hakeem (traditional healer). In retrospect this could have been used as a pointer towards pulmonary TB. However it seemed unlikely when considering the negative exposure history along with the $80+$ pack year history of smoking. This time around we were more likely and convinced while starting empiric ATT prior to the biopsy, based on our previous experience.

In both cases our patients had significant risk factors for both laryngeal TB and LCa. We present these cases to emphasize that although laryngeal tuberculosis presenting with primary laryngeal symptoms is a rarity, it must be considered when evaluating dysphagia or dysphonia in populations where TB is endemic.

\section{REFERENCES}

1. Topak M, Oysu C, Yelken K, Sahin-Yilmaz A, Kulekci M. Laryngeal involvement in patients with active pulmonary tuberculosis. Eur Arch Otorhinolaryngol. 2008;265:327-30.

2. Williams RG, Douglas-Jones T. Mycobacterium marches back. J Laryngol Otol. 1995;109:5-13.

3. Kim MD, Kim DI, Yune HY, Lee BH, Sung KJ, Chung TS, et al. CT findings of laryngeal tuberculosis: comparison to laryngeal carcinoma. J Comput Assist Tomogr. 1997;21:29-34.

4. Suhail A, Ahmed MS, Sobani ZU, Ghaffar S. Laryngeal tuberculosis presenting as laryngeal carcinoma. JPMA The Journal of the Pakistan Medical Association. 2012;62:167-8.

5. Country Profile: Pakistan; Global Tuberculosis Control. World Health Organization, 2003.

6. Bhurgri Y, Bhurgri A, Hassan SH, Zaidi SH, Rahim $A$, Sankaranarayanan $R$, et al. Cancer incidence in Karachi, Pakistan: first results from Karachi Cancer Registry. Int J Cancer. 2000;85:325-9. 